\title{
Suppression of broadband noise radiated by a low-speed fan in a duct $^{\text {a) }}$
}

\author{
L. Huang, ${ }^{\text {b) }}$ X. Ma, and L. G. Feng \\ Department of Mechanical Engineering, University of Hong Kong, Pokfulam Road, Hong Kong SAR, China
}

(Received 11 April 2009; revised 2 March 2010; accepted 3 March 2010)

\begin{abstract}
Attenuation of ducted fan noise remains a technical challenge in the low frequency range as traditional duct lining becomes ineffective. This study proposes a reactive method to suppress the sound radiation from an axial-flow fan. The method is particularly effective in the low frequency region and covers a broad band. Its effect is derived from two mechanisms. One is the reduction in the confining effects of duct walls when the duct radius is increased; the other is the acoustic interference between the direct radiation from the fan and reflections by the duct junctions. This interference is always destructive for axial dipoles when the frequency approaches zero. This performance differs from normal passive control methods, which become totally ineffective toward zero frequency. An approximate plane-wave theory explains the essential physics of the method, and its quantitative prediction is found to agree well with a full numerical simulation using a spectral method of Chebyshev collocation. The latter is validated by experiment using an axial-flow fan in a duct of finite length. Broadband noise reduction is achieved while the flow speed is kept unchanged. Practical difficulties of implementation for a fan with high pressure increase are discussed. (C) 2010 Acoustical Society of America. [DOI: 10.1121/1.3372749]
\end{abstract}

PACS number(s): 43.50.Gf, 43.50.Nm, 43.28.Ra [KVH]

Pages: $152-163$

\section{INTRODUCTION}

Fan noise abatement finds wide applications ranging from small computer cooling fans, vehicle and machinery cooling fans, and building ventilation fans, to large compressor and turbo-engine fans used in aircrafts. Among these applications, ventilation and aero-engine fans are ducted, while others are more open or only partially enclosed. In this study, we focus our attention on the ducted fan. Both theoretical and experimental investigations are involved. Although the numerical simulation method introduced in this study is applicable to radiation in higher order duct modes, the configuration of noise attenuation is most effective in the low frequency regime. Therefore the potential application of the study is limited to low-speed fans for the time being. Within such a framework, a very brief overview is now given for the fan noise mechanisms and methods of fan noise abatement. The method of the numerical modeling is introduced in Sec. II.

Fan noise has many sources. The basic mechanism is similar for many different kinds of fans, the simplest being an open propeller, which was studied by Gutin (1948). However, the so-called Gutin noise concerns the radiation by the steady loading on rotating blades, and it is normally insignificant for small fans with low tip Mach number. The general theory of aerodynamic sound was established by Lighthill (1952) in what is known as the "acoustic analogy," which was later extended by Curle (1955), and Ffowcs Williams and Hawkings (1969) to include the effects of station-

\footnotetext{
Part of this manuscript was presented at Inter-Noise 2008 in Shanghai in October, 2008

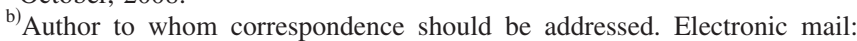
Lixi.Huang@hku.hk
}

ary and moving solid surfaces, respectively. The solid surfaces serve as "mirrors" of aerodynamic sources (quadrupoles), giving rise to dipoles distributed all over solid surfaces exerting unsteady forces on fluid. These dipoles, also called loading noise, are much more powerful in radiating sound at medium to low flow speeds. Unless there is a volume source such as unsteady heat release from combustion, dipole is the dominant feature in applications relevant to low speeds. In propeller or compressor analysis, a term called "thickness" noise is also encountered, and it is expressed in monopolelike integrals in the Ffowcs WilliamsHawkings equation. For machines with rigid surfaces, such source degenerates into dipole and is much weaker at low frequencies. Moreover, it does not radiate any propagating mode in a duct containing a fan (Goldstein, 1976). Another form of aerodynamic noise source modification by structure is the so-called trailing edge noise (Ffowcs Williams and Hall, 1970), which arises when turbulence is convected past an edge of semi-infinite plane. Trailing edge noise is insignificant when the structural surface dimension is compact compared with the wavelength.

The present study is exclusively focused on the noise radiated by the unsteady fluid loading on blades arising from (1) general rotor-stator interaction over the bulk of the blade span (Kemp and Sears, 1953, 1955), (2) tip leakage flow (Fukano et al., 1986; Dunne and Howe, 1997; Jang et al., 2003), or (3) non-uniform inlet flow interacting with the rotating blades (Trunzo et al., 1981). A real fan blade can be modeled as distributed dipole sources along its radial span, including the tip region, and the peak source strength is often close to the blade tip. In this study, however, the radial distribution of the sources is not the most important factor for the low frequency radiation from the baseline case of an 
(a)
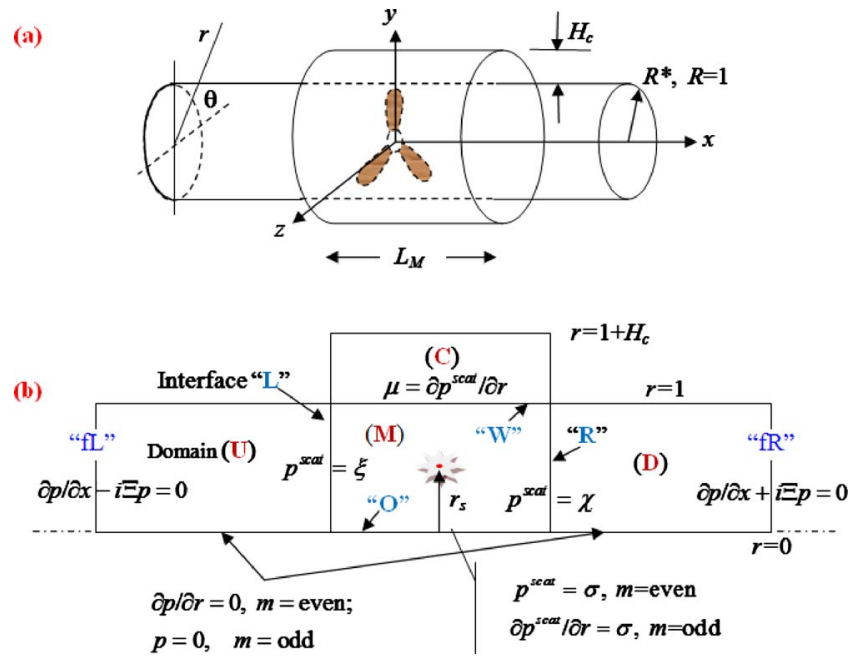

FIG. 1. (Color online) Physical and computational model of fan noise radiation in a circular duct: (a) three dimensional view of a fan installed in a duct with a segment with expanded casing (the main duct radius $R^{*}$ is used as a length normalization scale) and (b) all the boundary conditions and domain/interface labels for computation in the plane of duct axis and radius.

axial-flow fan installed inside a uniform duct. In this case, a single dipole for one blade can be used. For higher frequencies, however, the effect of the radial position of the concentrated sources should be looked at when acoustic casing treatment is introduced. Acoustic casing treatment is here defined as the enlarged duct cross section around a fan with or without porous material filling in the expanded volume, shown in Fig. 1(a). Note that in the turbomachinery aerodynamics literature (Hill et al., 1998), casing treatment normally implies shallow casing recess or slots created for the purpose of improving the flow condition around the tip region for either efficiency gain or the extension of stable operation range. There is no reason to believe that these two kinds of casing treatments are totally unrelated but the focus of the present study is on acoustics.

For ducted fans, sound waves radiated by various sources experience the confining effects of duct walls and their propagation is governed by duct acoustics modes (Tyler and Sofrin, 1962). The plane-wave mode is the only possible mode when the frequency is below the first circumferential mode cut-on, which is around $0.2928 c_{0}^{*} / R^{*}$, where $c_{0}^{*}$ is the speed of sound and $R^{*}$ is the duct radius. For example, for the $133.5-\mathrm{mm}$, diameter duct used in the current experiment, the cut-on frequency is $f^{*}=1493 \mathrm{~Hz}$. The unsteady loading is divided into two components: one along the duct and another in the circumferential direction. They are customarily called the thrust and drag noise, respectively. The lowest propagating mode for the circumferential loading (drag noise) is the first mode with the normalized frequency $f$ $=f^{*} R^{*} / c_{0}^{*}$ above 0.2928 . Below this frequency, the only radiating force component for the plane-wave mode is the thrust force. The sound radiated by this force has anti-phase relation at the two sides of the source. When the radiated sounds from the two sides are brought together by acoustic reflections, destructive interference is expected to occur. This is the basic motivation for the current study. The result can also be considered from the point of view of modification of system radiation impedance.

A few words about the existing noise abatement practice may be necessary here. Passive control remains to be the preferred engineering solution for fan noise. For obvious reasons of preserving the smooth flow passage, duct lining is the main configuration in design (Motsinger and Kraft, 1995). Having cited this classical volume edited by Hubbard, it must be said that the major technical difficulties for large aero-engines and small ventilation fans are different. The former typically has higher order modes, for which modal scattering and sound penetration into the lining is a major concern (McAlpine et al., 2006), while the latter may have strong low frequency components for which the sound absorption is ineffective. Active control has also been studied for fan noise for both free field and ducted fan applications. There are commonalities between active fan noise control and active control of other noise sources, such as the need to remove feedback from secondary sources (Prezelj and $\breve{C u}$ dina, 2007). But one of the main differences lies in the effort, made by researchers working on fan noise, to match the modal or spatial structure of the secondary source with that of the primary noise. For example, Lauchle et al. (1997) applied axial shaking of the fan rotor, while Schulten (2001) shook the vanes. Neuhaus et al. (2003) used air jet around the casing to create the same acoustic mode as the rotorstator interaction in order to control the propagating second order mode, while Namba and Murahashi (2008) investigated theoretically the effectiveness of flush mounted pistons to control the noise created by the inlet flow gust and rotor. Wang and Huang (2006) controlled the drag noise by driving four loudspeakers on the back of the fan motor with tachometer signals of the motor and its time derivative for perfect match of temporal and spatial signature of the rotor-strut interaction, having previously succeeded in tackling the easier problem of thrust noise by loudspeakers driven at the same phase (Wang et al., 2005). In most of these active controls, the main objective is to reduce the blade-passing frequency and a few of its harmonics, while the reduction in other broadband frequency components would have to come through the improvement of aerodynamic conditions such control measures may bring as a by-product. The current study is passive control in nature but the spirit of creating the destructive acoustic interference is the same as active control. The most perfect match for the fan noise must be the fan noise itself, leading to this investigation of the effectiveness of the self-cancellation between the noise radiated toward the upstream and that toward the downstream. The objective is not limited to tonal noise; it covers the whole frequency band below the first cut-on frequency of the duct.

In what follows, Sec. II briefly describes the numerical procedure for the simulation of a simplified fan noise radiation in a duct with casing treatment. Section III analyzes the predicted sound power with various forms of casing treatment for a shallow casing treatment. It is then followed by a plane-wave theory, which explains the main features of the full numerical results. Perfect match is achieved toward the $\mathrm{dc}$ frequency, and this formulation reveals the crucial mecha- 
nism of acoustic interference in the minimization of dipole noise radiation with a compact cavity. Section IV introduces experimental validation of theory, which is necessarily conducted in a duct of finite length and also with a deeper cavity. About $10 \mathrm{~dB}$ noise attenuation is achieved for most frequencies below the higher order mode cut-on, but some frequencies see negative insertion loss, which also agrees with the prediction. Potential issues related to the actual implementation of the concept introduced in this study are also discussed in this section. Conclusions are drawn in Sec. V.

\section{NUMERICAL METHOD}

In this study, the focus is on whether the general dipole source characterizing a ducted fan can be weakened in its ability to radiate noise. The theme of prediction is therefore one of sound radiation and propagation instead of aerodynamic source strength. The latter would have required computational fluid dynamics to describe the unsteady flow pressure on blades and other load-bearing surfaces. There are efforts to combine unsteady fluid dynamics and acoustics into a one-step prediction, but that is a new endeavor for which the low-speed fan may not be the most useful application. The traditional concept of acoustic analogy is therefore retained.

One of the main difficulties arising from the ducted fan configuration is the effect of the confining walls. This effect can be neglected when dealing with high frequency fan noise radiation, such as in Dunne and Howe (1997). For low to medium frequencies, however, more elaborate methods have to be employed in order to avoid integration over duct walls. Green's function for infinite duct can be used (Lewy, 2007) in place of free-space Green's function in the Ffowcs Williams-Hawkings equation if the end reflection can be ignored. This is not the case in the present study. Moreover, casing treatment with or without bulk reactive absorption is better dealt with by a full numerical method although, for this particular case, a more traditional, semi-analytical approach of modal decomposition and mode-matching (Eversman, 1995) can certainly be used. One possible fully numerical approach is the boundary element method (BEM) (Dunn et al., 1999; Choi and Lee, 2006). BEM is particularly suitable for radiation into open space but its advantage over methods such as finite element method in internal problems diminishes with the amount of the required mesh. In this study, the spectral method of Chebyshev collocation with domain decomposition, which is really a prototype spectral element method (Karniadakis and Sherwin, 2005), is employed. As shown below, the use of the dipole source simulating the fan noise source implies that the end result of the numerical simulation is simply a Green's function, taking into account the rigid-wall boundary condition and the impedance condition at the finite duct inlet and exit. With the acoustic field calculated at these duct openings, the sound power output is calculated as the main result for analysis.

\section{A. Governing equation and boundary conditions}

The configuration to be considered is shown in Fig. 1(a) in three dimensional view and in Fig. 1(b) in the plane of duct axis $x$ and radius $r$. A segment of tube expansion is provided to reduce the fan noise radiation. When the radius of the central duct $R^{*}$ is used as the normalization scale, its dimensionless radius becomes unity $(R=1)$. The dimensionless cavity depth is $H_{c}=H_{c}^{*} / R^{*}$ and the length is $L_{M}$ $=L_{M}^{*} / R^{*}$. Superscript asterisks are used to denote dimensional quantities, while its dimensionless counterparts adopt the same symbols without asterisks. Speed of sound $c_{0}^{*}$ will be used for velocity scale, so that the dimensionless frequency is defined as $f=f^{*} R^{*} / c_{0}^{*}$. Pressure is normalized by $\rho_{0}^{*}\left(c_{0}^{*}\right)^{2}$, where $\rho_{0}^{*}$ is the air density.

As shown in Fig. 1(b) for computational purposes, an upstream domain $(U)$ and downstream domain $(D)$ are created, while the middle domain is labeled as $(M)$ and cavity $(C)$. The far left " $\mathrm{fL}$ " and far right "fR" interfaces are the open ends of the finite duct where proper radiation boundary conditions are applied. They can also be an arbitrary truncation of computational domain for an infinite duct where perfectly outgoing wave condition is enforced. The flow velocity is assumed to be very low (about $3 \mathrm{~m} / \mathrm{s}$ in experiment) and convection effects are thus ignored. When Mach number reaches a reasonable value, such as 0.2 , it is expected to have impacts on results but that is left to future studies. The problem is cast in the frequency domain, and the governing inhomogeneous Helmholtz equation is

$$
\left(\frac{\partial^{2}}{\partial x^{2}}+\frac{\partial^{2}}{\partial r^{2}}+\frac{\partial}{r \partial r}+\frac{\partial^{2}}{r^{2} \partial \theta^{2}}+k^{2}\right) p=\nabla \cdot[\boldsymbol{F} \delta(\boldsymbol{x}-\boldsymbol{y})],
$$

where $\boldsymbol{F} e^{i \omega t}$ is the concentrated force exerted on the fluid, $\omega$ is the angular frequency, $k=\omega / c$ is the source wavenumber, and $\boldsymbol{x}$ and $\boldsymbol{y}$ are vectors for the observer and the source, respectively. Fourier transformation in the circumferential direction of the circular duct $\theta$ is applied first. The sound pressure is written as

$$
p=\sum_{m=-\infty}^{+\infty} P_{m} e^{i m \theta}, \quad P_{m}=\frac{1}{2 \pi} \int_{0}^{2 \pi} p e^{-i m \theta} d \theta .
$$

Since each mode $m$ is dealt with at one step in the solution, for which the operator of $\partial^{2} / \partial \theta^{2}$ in Eq. (1) becomes $-m^{2}$, the modal amplitude $P_{m}$ is re-written as $p$ in subsequent discussions for simplicity unless specific reference to mode index $m$ is needed for clarification. As is described below, the solution method for the $x$-r plane will be Chebyshev collocation, or pseudo-spectral method, but the full method is more appropriately described as Fourier-Chebyshev spectral method.

The boundary condition on the hard walls is well known

$$
\partial p / \partial n=0,
$$

and that on the central line $(r=0)$ simply derives from the circumferential modal structure

$$
P_{m}=0 \quad(m=\text { odd }), \quad \partial P_{m} / \partial r=0 \quad(m=\text { even }) .
$$

The outgoing wave condition for a plane-wave is simply $i k p+\partial p / \partial n=0$, where $n$ is the local outward normal direction. If traveling or decaying higher order modes in the radial direction are present, the boundary condition is given by the 
parabolic wave equation. The wave equation can be rewritten as $\left(\partial_{x}^{2}+\Xi^{2}\right) p=0$, where $\Xi$ is a pseudo-differentiation operator defined below as

$$
\Xi=\sqrt{k^{2}+\frac{\partial^{2}}{\partial r^{2}}+\frac{1}{r} \times \frac{\partial}{\partial r}-\frac{m^{2}}{r^{2}}} .
$$

This simplifies to wavenumber $k$ for a plane-wave mode in the duct. The wave equation can thus be factorized into two parts

$$
\left(\partial_{x}^{2}+\Xi^{2}\right) p=\left(\partial_{x}+i \Xi\right)\left(\partial_{x}-i \Xi\right) p=0,
$$

one traveling to the right $\left(\partial_{x}+i \Xi\right) p=0$, and another to the left $\left(\partial_{x}-i \Xi\right) p=0$, which can be combined as

$$
\left(\partial_{n}+i \Xi\right) p=0,
$$

where the outward normal direction $n$ is $+x$ for the right and $-x$ for the left.

In the current spectral element computation, the derivatives in this square-root operator $\Xi$ are represented by matrices (Boyd, 2001) and $\Xi$ is computed by matrix diagonalization with the help of eigenvalue decomposition. Detailed procedure for this operator is given in (Huang, 2008). When the problem is discretized, the column vector of pressure gradient $\partial p / \partial x$ at the far right exit is denoted as $\left[\partial_{x} p\right]_{(f R)}$, which is the transpose of vector $\left[\partial_{x} p_{j=0} \cdots \partial_{x} p_{j=N_{r}}\right]_{(f R)}$, where $N_{r}$ is the number of Gauss-Lobatto segments in the radial mesh from $r=0$ to $r=1$

$$
r_{j}=\frac{1}{2}\left[1-\cos \left(j \pi / N_{r}\right)\right] \quad j=0,1,2, \ldots, N_{r} .
$$

Note that square brackets are used to denote a vector or a matrix sampled over the mesh grids for the enclosed variable unless a new symbol is reserved for use in the discrete sense only, such as $\xi$ below. Boundary condition Eq. (5b) is written in a matrix form as follows for the far left and far right boundaries

$$
\left[\partial_{x} p\right]_{(f L)}=[i \Xi][p]_{(f L)}, \quad\left[\partial_{x} p\right]_{(f R)}=[-i \Xi][p]_{(f R)} .
$$

When the far left and far right ends are duct openings, with or without flange, complex wave scattering occurs together with radiation into the far field. For frequencies above the first cut-on frequency, a matrix impedance can be defined and developed, either in the form of modal coefficients, or in a collocation style. An example of such work for rectangular opening is given by Pierce et al. (2002). Such matrix would replace the matrix of $[\Xi]$ above. For the experimental work performed in the current study, attention is focused on the plane-wave mode, for which a lumped parameter (scalar) impedance suffices. In this case, the boundary condition becomes

$$
\left(i k / Z_{\text {open }}+\partial / \partial n\right) p=0, \quad Z_{\text {open }}=p^{*} /\left.\left(\rho_{0}^{*} c_{0}^{*} u_{n}^{*}\right)\right|_{\text {open end }},
$$

where the dimensionless open-end impedance $Z_{\text {open }}$ can be measured in experiment, or calculated from classical theories as a function of frequency (Morse and Feshbach, 1953, Eq. 11.4.47). Impedance $Z_{\text {open }}$ is replaced by unity for the boundaries of "fL" and "fR" in an infinite duct. In both cases, the matrix $[\Xi]$ in Eq. (5c) is replaced by $k / Z_{\text {open }}$ times the unit diagonal matrix.

\section{B. Pressure decomposition and solution procedure}

The solution procedure specific to the four-domain configuration shown in Fig. 1(b) is briefly described below. First of all, the sound pressure is divided into two parts: free-space radiation by the source dipoles, indicated by a superscript "rad," and the scattering by all boundaries, indicated by a superscript "scat"

$$
p=p^{\mathrm{rad}}+p^{\mathrm{scat}} .
$$

The radiation pressure $p^{\mathrm{rad}}$ is given further below. The scattering pressure $p^{\text {scat }}$ should satisfy the homogeneous version of wave equation [Eq. (1)]. All boundary conditions are written for the full pressure $p$. The calculation of the radiation field is limited to the middle domain labeled as $(M)$ in Fig. 1(b), while the pressure for three other domains $(U),(C)$, and $(D)$ is calculated for the full pressure directly without being divided into the radiation and scattered parts.

Since the problem is linear, the method of influence matrix can be used. Denote the values of the scattered pressure at the left interface " $L$ " in domain $(M)$ by a column vector $\xi$, those at " $R$ " and " $W$ " by $\chi$ and $\mu$, respectively

$$
\xi=\left[\begin{array}{c}
p_{j=0}^{\text {scat }} \\
\vdots \\
p_{j=N_{r}}^{\text {scat }}
\end{array}\right]_{L} \quad \chi=\left[\begin{array}{c}
p_{j=0}^{\text {scat }} \\
\vdots \\
p_{j=N_{r}}^{\text {scat }}
\end{array}\right]_{R}, \quad \mu=\left[\begin{array}{c}
p_{j=0}^{\text {scat }} \\
\vdots \\
p_{j=N_{x}}^{\text {scat }}
\end{array}\right]_{W},
$$

where subscripts " $L$," " $R$," and " $W$ " indicate the boundary locations, and $N_{x}$ is the number of axial Gauss-Lobatto segments for domain $(M)$. These are the unknowns to be determined through matching conditions across the interfaces before the full field solution is obtained. The unknown to be determined on the central line " $O$ " is denoted as $\sigma$, which is taken to be the complex amplitude for the scattered pressure for even mode $m$ or pressure gradient for odd mode $m$

$$
\sigma=\left[\begin{array}{c}
P_{m, j=0}^{\mathrm{scat}} \\
\vdots \\
P_{m, j=N_{x}}^{\text {scat }}
\end{array}\right]_{O} \quad(m=\text { even }), \quad \sigma=\left[\begin{array}{c}
\partial_{r} P_{m, j=0}^{\mathrm{scat}} \\
\vdots \\
\partial_{r} P_{m, j=N_{x}}^{\text {scat }}
\end{array}\right]_{O} \quad(m=\text { odd }) .
$$

Note that the boundary condition to be satisfied on boundary " $O$ " is $\partial_{r} P_{m}=0$ for even $m$ and $P_{m}=0$ for odd $m$, which is listed below in Eq. (9).

An influence matrix is denoted by a subscript for the evaluation location and a superscript for the source of influence. For example, the pressure gradient at the right interface " $R$ " caused by the left interface " $L$ " is calculated by the product of the influence matrix $\left(\partial_{x} p\right)_{R}^{L}$ with the column vector $\xi$, in which the $j$ th column of the influence matrix $\left(\partial_{x} p\right)_{R}^{L}$ is the result of a unit perturbation at the $j$ th entry of $\xi$ when all other entries are zero, and appropriate homogeneous boundary conditions are used for all other boundaries. Since each interface in domain $(M)$ is influenced by all four bound- 
aries in the domain, including the interface itself, the actual pressure value at each interface is calculated as the sum of influences from all four boundaries, e.g.

$\left(\partial_{x} p\right)_{R}=\left(\partial_{x} p\right)_{R}^{L} \xi+\left(\partial_{x} p\right)_{R}^{R-} \chi+\left(\partial_{x} p\right)_{R}^{O} \sigma+\left(\partial_{x} p\right)_{R}^{W} \mu+\left(\partial_{x} p\right)_{R}^{\mathrm{rad}}$,

where the radiation contribution is indicated by the superscript "rad" and " $R-$ " indicates the left-hand side of the interface " $R$." Meanwhile, the pressure gradient on the righthand side of interface " $R$ " is calculated by the downstream domain $(D)$, hence

$$
\left(\partial_{x} p\right)_{R}=\left(\partial_{x} p\right)_{R}^{R+}\left(\chi+p_{R}^{\mathrm{rad}}\right) .
$$

Note that $p_{R}^{\text {rad }}$ is included since the downstream domain solves only for the full pressure. The equality of pressure gradient $\left(\partial_{x} p\right)$ at the two sides of interface " $R$ " provides the necessary boundary condition for " $R$." The pressure continuity is already served by having the same scattered pressure $\chi$ for both sides of " $R$." The four coupling or boundary conditions at " $L$, ," $R$, , " $O$, ," and " $W$ " are listed below

$$
\begin{aligned}
& \left(\partial_{x} p\right)_{L}^{\mathrm{rad}}+\left(\partial_{x} p\right)_{L}^{L+} \xi+\left(\partial_{x} p\right)_{L}^{R} \chi+\left(\partial_{x} p\right)_{L}^{O} \sigma+\left(\partial_{x} p\right)_{L}^{W} \mu \\
& \quad=[i \Xi]\left(\xi+p_{L}^{\mathrm{rad}}\right), \\
& \left(\partial_{x} p\right)_{R}^{\mathrm{rad}}+\left(\partial_{x} p\right)_{R}^{L} \xi+\left(\partial_{x} p\right)_{R}^{R-} \chi+\left(\partial_{x} p\right)_{R}^{O} \sigma+\left(\partial_{x} p\right)_{R}^{W} \mu \\
& \quad=[-i \Xi]\left(\chi+p_{R}^{\mathrm{rad}}\right), \\
& X_{O}^{\mathrm{rad}}+X_{O}^{L} \xi+X_{O}^{R} \chi+X_{O}^{O} \sigma+X_{O}^{W} \mu=0 \quad \text { where } X \\
& \quad \equiv\left[\partial_{x} P_{m}\right](m=\text { even }) \text { and } X \equiv\left[P_{m}\right](m=\text { odd }), \\
& \left(\partial_{r} p\right)_{W}^{\mathrm{rad}}+\left(\partial_{r} p\right)_{W}^{L} \xi+\left(\partial_{r} p\right)_{W}^{R} \chi+\left(\partial_{r} p\right)_{W}^{O} \sigma+\left(\partial_{r} p\right)_{W}^{W} \mu \\
& \quad=\left(\partial_{r} p\right)_{W}^{W+} \mu .
\end{aligned}
$$

Note that in the first two equations of Eq. (9), the pseudodifferentiation matrix $[\Xi]$ should be replaced by $k / Z_{\text {open }}$ times the unit diagonal matrix when an open end with dimensionless impedance $Z_{\text {open }}$ is simulated instead of a pure outgoing wave boundary. Equation (9) can be solved for $\xi, \chi, \mu, \sigma$, and subsequently, all acoustic fields are calculated and assembled.

\section{Source term analysis}

Before the computation for the scattered field begins, the free-space radiation terms are calculated for all four sides of domain $(M)$, e.g., $p_{R}^{\text {rad }}$, with contributions from all dipole sources. The results then undergo Fourier transform in circumferential direction. It is assumed that the rotor-stator interaction is the noise source, and the sound from $S$ stator blades is to be calculated. Note that sound radiation from rotor blade carries the Doppler effect and will add unnecessary complication for the purpose of the current study. The unsteady force experienced by a stator has the fundamental blade-passing frequency of $B \times \mathrm{rps}$, where rps is given in revolutions per second, and $B$ is the number of rotor blades. For the $n_{h}$ th harmonic, the forces experienced by stator index $j$ leads that of $j=0$ by a phase angle of $n_{h} B(2 \pi) j / S$ when the blade rotates clockwise, while $\theta$ and index $j$ are measured anti-clockwise

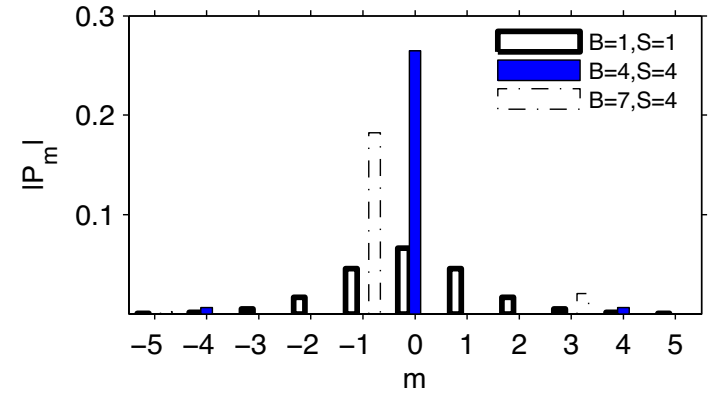

FIG. 2. (Color online) Effect of dipole source distributions for the freespace radiation for three sets of rotor (B) and stator (S) blade numbers, with observer at $x=2, r=1$ and frequency $f=0.333$.

$$
\left.\begin{array}{c}
p^{\mathrm{rad}}=\sum_{j=0}^{S-1} p_{j}^{\mathrm{rad}}, \quad p_{j}^{\mathrm{rad}}=\frac{i k F_{\hat{r}_{j}}}{4 \pi \hat{r}_{j}}\left(1+\frac{1}{i k \hat{r}_{j}}\right) \exp \left(-i k \hat{r}_{j}\right), \\
\hat{r}_{j}=\boldsymbol{x}(x, r, \theta)-\boldsymbol{y}_{j}, \quad F_{j}=F_{0} e^{i n_{h} B(2 \pi) j / S}, \\
P_{m}^{\mathrm{rad}}=\frac{1}{2 \pi} \int_{0}^{2 \pi} p^{\mathrm{rad}} \exp (-i m \theta) d \theta
\end{array}\right\}
$$

Here, $\boldsymbol{y}_{j}$ is the position of the dipole of index “ $j$," $\boldsymbol{x}(x, r, \theta)$ is the observer position on interfaces such as " $R$ " or any grid point in domain $(M), \hat{r}_{j}$ is the vector distance between the observer and the $j$ th source, and $F_{\hat{r}_{j}}$ is the source strength $\boldsymbol{F}_{j}$ projected on $\hat{r}_{j}$. In this study, Clenshaw and Curtis (1960) integration formula is used with the Gauss-Lobatto grid. Note that the amplitude of force on each blade, written as $F_{0}$ in Eq. (10), is assumed to be unity. In other words, $P_{m}^{\mathrm{rad}}$ calculated this way is actually normalized by a pressure equal to the actual force divided by $\left(R^{*}\right)^{2}$.

Figure 2 shows the modal amplitude bar charts for three sets of rotor-stator blade number combinations: $(B, S)$ $=(1,1),(4,4)$, and $(7,4)$. The first blade passing frequency of $f=0.333$ is used but the amplitudes shown are for the freespace radiation with an observer position of $x=1$ and $r=1$. For the first set of $(B, S)=(1,1)$, significant modal amplitude spreads over $m \in(-5,+5)$, but later calculation for the sound power of in-duct radiation shows that there is only power output for the mode of $m=0$ and \pm 1 due to modal cutoff for higher order modes. The lowest cut-on dimensionless frequency of 0.2928 occurs for the first radial mode of $n=1$ for $m= \pm 1$. Note that positive and negative signs matter in this case due to the fan rotation, and the summation of modes is over $m=-\infty \rightarrow+\infty$ instead of $m=0 \rightarrow+\infty$. The second set of $B=S=4$ is a "resonance" condition, and its modal pressure distribution is only over multiples of 4 for obvious reasons. In this case, only the plane-wave mode of $m=n=0$ will be radiated in a duct. The third set is for a real fan design with $B=7$ and $S=4$. This design avoids the "resonance" condition of leading order noise radiation by the thrust force, $m=0$, and the next low order mode is $m=-1$. This is a high-order (weak) mode for the thrust force radiation, but it becomes the leading order (strong) radiation for the circumferential force (Huang, 2003), which is absent in this example. This is so because the circumferential force changes direction once per cycle. The accuracy of the code for the cut-on modes is 
validated against Green's function for a single dipole in a rigid duct. Details of the validation is given in Huang and Cheung (2008) for the more complex configuration of a circumferential dipole with $m=1$. It is also shown there that the deviation of the calculated sound power $W$ from the analytical value $W_{0}$ for cut-on modes is generally small $\left(\mid W / W_{0}\right.$ $\left.-1 \mid<10^{-7}\right)$.

\section{NUMERICAL RESULTS AND PLANE-WAVE APPROXIMATION}

To focus on the mechanism of sound-casing interaction, the subsequent computations are conducted for $B=S=4$. In the experimental studies, a fan with nine blades will be used, but it is easily shown that the number of blades in such coincident mode of radiation does not influence the conclusions. As shown by Fig. 2, the only radiating mode is the plane-wave mode of $m=n=0$. Higher radial mode of $n=1$ is cut on when the frequency is above 0.6096. Calculations in this section are cut off at $f=0.6$.

\section{A. Sound radiation from four configurations}

The variation in sound power radiated by the axial dipoles in four casing configurations is studied by the numerical method described in Sec. II. The first is the baseline configuration of rigid duct. The second is a duct with empty casing treatment, which is essentially an expansion chamber. The third is a locally reactive lining, for which the impedance boundary condition is set as

$$
\begin{aligned}
& \left(i k / Z_{\mathrm{sam}}\right) p+\partial p / \partial r=0, \quad Z_{\mathrm{sam}}=\rho_{\mathrm{sam}} c_{\mathrm{sam}} \operatorname{coth}\left(\Gamma_{\mathrm{sam}} H_{c}\right), \\
& \rho_{\mathrm{sam}}, c_{\mathrm{sam}}, \Gamma_{\mathrm{sam}}=\operatorname{func}\left(f \rho_{0} / R_{\mathrm{sam}}\right), \quad R_{\mathrm{sam}}=R_{\mathrm{sam}}^{*} R^{*} /\left(\rho_{0}^{*} c_{0}^{*}\right),
\end{aligned}
$$

where $H_{c}$ is the cavity depth, $R_{\text {sam }}^{*}$ and $R_{\text {sam }}$ are the dimensional and normalized flow resistivities, respectively, and $\rho_{\text {sam }}, c_{\text {sam }}$, and $\Gamma_{\text {sam }}$ are the complex equivalent fluid density, speed of sound, and propagation constant for porous sound absorption materials, respectively, which are taken from the empirical data of Delany and Bazley (1970). All normalized properties of sound absorption material are functions of a frequency variable $f^{*} \rho_{0}^{*} / R_{\text {sam }}^{*}$. The last type of casing is the bulk reactive lining for which the transverse acoustic particle velocity and pressure are continuous across the interface at $r=1$. The calculation is conducted for different fan rotational speeds, and only the first blade-passing frequency radiation is analyzed. In other words, the spectral plots shown below are not the spectra for a single fan rotating at a given speed, but rather the blade-passing frequency radiation for the same fan rotating at different speeds. The conclusions drawn regarding the effect of casing treatment shall be valid for the normal case of fan noise radiation under a single rotational speed, in which radiation other than blade-passing frequency is expected to be caused by non-deterministic events of rotor-stator interaction. This assumption is fully validated in experiment described in Sec. IV.

The spectra of sound radiation power $W$ are given in Fig. 3. The configuration of rigid wall (thick solid line) has a constant sound power. This is so because the point axial

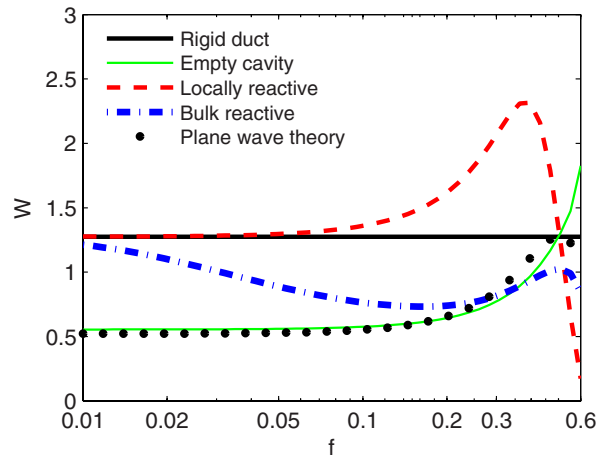

FIG. 3. (Color online) Radiated sound power for axial dipoles of unit force amplitude $(B=S=4)$, with four different casing treatments. The dimensionless flow resistivity for the sound absorption material used in the two linings is $R_{\text {sam }}=0.5$. The cavity length is $L_{M}=1$ and the depth is $H_{c}=0.25$. The plane-wave theory is for the empty cavity.

force $(F)$ radiation is transformed into plane waves of opposite phase in the two sides of the duct with a sound pressure amplitude of $\hat{p}=F /\left(2 \pi R^{2}\right)$. For this example, the force is $F$ $=1$ for each of the four dipoles, so the dimensionless sound power is found to be $4 / \pi$, as shown below

$$
\begin{aligned}
& \hat{p}=\frac{(4 F)}{2 \pi R^{2}}=\frac{2}{\pi}, \quad I_{\mathrm{rms}}=\frac{1}{2} \hat{p}^{2}=\frac{2}{\pi^{2}}, \\
& W=2 I_{\mathrm{rms}}\left(\pi R^{2}\right)=\frac{4}{\pi} .
\end{aligned}
$$

The results for the empty cavity (thin solid line) are much below the baseline value of $4 / \pi$ over most of the frequency range except that the resonancelike peak appears when $f$ approaches 0.6. The locally reactive lining (dashed line) stays above the baseline result for most of the low frequencies, and it goes below the baseline level at $f=0.50$. The bulk reactive lining is better than the baseline, but is worse than the empty cavity for most low frequencies. Notice that at very low frequencies of 0.01 , sound absorption material is much like a solid wall, so that both locally reactive and bulk reactive linings converge toward the baseline result. The filled dots are for the plane-wave theory for the configuration with empty cavity, which is described in the latter part of Sec. III B.

\section{B. Approximate plane-wave theory}

The good performance of the empty casing treatment down to the dc frequency, as shown by the thin solid line in Fig. 3, suggests that the mechanism is fundamentally one of low frequency. At the low frequency limit $(f \rightarrow 0)$, the dipole radiation is rapidly turned into plane waves. For a uniform duct of radius $R$, Green's function for an axial dipole placed at $\left(x_{1}, r_{1}, \theta_{1}\right)$ is (Morse and Ingard, 1968)

$$
\begin{aligned}
& G\left(r, \theta \mid r_{1}, \theta_{1}\right) \\
& \quad=\sum_{m, n=0}^{\infty} \frac{J_{m}\left(k_{m n} r_{1}\right) J_{m}\left(k_{m n} r\right) \cos \left[m\left(\theta-\theta_{1}\right)\right] e^{-i k_{x m n}\left|x-x_{1}\right|}}{\left(1+\delta_{0, m}\right) \pi R^{2}\left(1-m^{2} / k_{m n}^{2}\right) J_{m}^{2}\left(k_{m n}\right)},
\end{aligned}
$$

where $k_{m n}$ is the wavenumber for mode " $m n$." For very low 
frequencies, only the plane wave of mode $m=n=0$ propagates $k_{00}=0, J_{0}(0)=1$; hence, $|G|=1 /\left(2 \pi R^{2}\right)$. The waves have opposite phase in the two directions. When these waves encounter the junctions, a pair of traveling waves is formed. The overall wave system is shown in Fig. 4 for a dipole placed at the axial center $x=0$ of the large duct, with a force $F$. The waves radiated to the left $(-)$ and right $(+)$ sides of the source are

$$
p_{-}=\frac{-F}{2 A_{2}} \exp [i k x], \quad p_{+}=\frac{+F}{2 A_{2}} \exp [-i k x],
$$

where $A_{2}$ is the cross section with the casing cavity. Defining the source pressure and area

$$
\begin{aligned}
& p_{s}=F /\left(2 \pi R^{2}\right), \quad A_{1}=\pi R^{2}=\pi, \\
& A_{2}=\pi\left(1+H_{c}\right)^{2}=\alpha A_{1} .
\end{aligned}
$$

The standing waves at the two sides of the source are

$$
\begin{array}{ll}
\text { source: } & p= \pm \alpha^{-1} p_{s} \exp [\mp i k x], \\
x<0: & p=A \exp (-i k x)+\left(B-\alpha^{-1} p_{s}\right) \exp (i k x), \\
x>0: & p=\left(A+\alpha^{-1} p_{s}\right) \exp (-i k x)+B \exp (i k x) .
\end{array}
$$

The pressure and volume flow continuity at the two junctions of $|x|=L_{M} / 2$ require

$$
\begin{aligned}
& x=-L_{M} / 2: \quad A e^{i k L_{M} / 2}+\left(B-\alpha^{-1} p_{s}\right) e^{-i k L_{M} / 2}=C e^{-i k L_{M} / 2}, \\
& \alpha\left[A e^{i k L_{M} / 2}-\left(B-\alpha^{-1} p_{s}\right) e^{-i k L_{M} / 2}\right]=-C e^{-i k L_{M} / 2}, \\
& x=+L_{M} / 2: \quad\left(A+\alpha^{-1} p_{s}\right) e^{-i k L_{M} / 2}+B e^{i k L_{M} / 2}=D e^{-i k L_{M} / 2}, \\
& \alpha\left[\left(A+\alpha^{-1} p_{s}\right) e^{-i k L_{M} / 2}-B e^{i k L_{M^{\prime}} / 2}\right]=D e^{-i k L_{M^{\prime}} / 2},
\end{aligned}
$$

which can be solved for the amplitudes of the two outgoing waves

$$
\begin{aligned}
& C=-2 p_{s} e^{i k L_{M}} \frac{(1-\alpha)+(1+\alpha) e^{i k L_{M}}}{(1+\alpha)^{2} e^{2 i k L_{M}}-(1-\alpha)^{2}}, \\
& D=2 p_{s} e^{i k L_{M}} \frac{(1-\alpha)+(1+\alpha) e^{i k L_{M}}}{(1+\alpha)^{2} e^{2 i k L_{M}}-(1-\alpha)^{2}} .
\end{aligned}
$$

The total power radiated is

$$
\begin{aligned}
W & =\frac{A_{1}}{2}\left(|C|^{2}+|D|^{2}\right) \\
& =8 W_{1} \frac{\left(\alpha^{2}+1\right)-\left(\alpha^{2}-1\right) \cos \left(k L_{M}\right)}{(\alpha-1)^{4}+(\alpha+1)^{4}-2\left(\alpha^{2}-1\right)^{2} \cos \left(2 k L_{M}\right)},
\end{aligned}
$$

where

$$
W_{1}=\left.W\right|_{\alpha=1}=A_{1}\left|p_{s}\right|^{2}
$$

is the sound power radiated in a rigid, uniform duct of cross section $A_{1}$. The cosine functions in Eq. (17a) can be evaluated for special chamber length

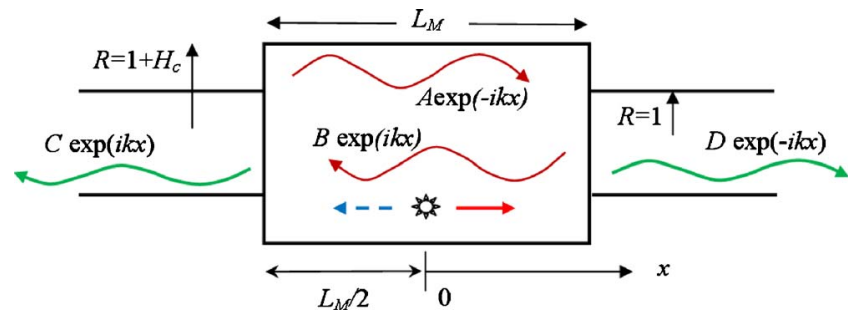

FIG. 4. (Color online) Plane wave theory for a dipole with force $F$ placed inside an expansion chamber of length $L_{M}$. The upstream and downstream regions are semi-finite.

$$
\frac{W_{1}}{W}=\left\{\begin{array}{l}
\alpha^{2}, \quad k L_{M}=2 n \pi \\
1, \quad k L_{M}=(2 n+1) \pi \\
\left(1+\alpha^{2}\right) / 2, \quad k L_{M}=(2 n+1) \pi / 2
\end{array}\right.
$$

where $n$ is any integer. If an insertion loss defined as $10 \log _{10}\left(W_{1} / W\right) \mathrm{dB}$ is plotted, as is done in Fig. 6(c) with the solid line, peak performance is found at the dc frequency $(k=0)$ and when the chamber is exactly a multiple of a full wavelength. No change is found $\left(W=W_{1}\right)$ when the chamber is an odd multiples of half wavelength. Intermediate results appear when the chamber length is any odd multiples of quarter wavelength. These results contrast with the transmission loss through the expansion chamber for a plane incident wave from outside the chamber. In the latter case, multiples of half wavelength has zero transmission loss and odd multiples of the quarter wavelength has the peak performance, with sound intensity ratio of $1+\left(\alpha-\alpha^{-1}\right)^{2} / 4$. When this peak ratio is compared with the peak power value of $\alpha^{2}$ given in Eq. (17c), the difference is a factor of 4.0 when the area ratio $\alpha$ is large. In other words, the dipole control inside an expansion chamber is typically $6.0 \mathrm{~dB}$ better off for the same expansion ratio $\alpha$.

The most important feature for the dipole control is that it has a peak performance at the dc frequency. The origin of the power ratio $W_{1} / W_{\mathrm{dc}}=\alpha^{2}$ is now analyzed in more details. If the chamber is extended to infinity, the sound power is reduced as well. The ratio of $W$ to such expansion reveals the contribution of the sound reverberation inside the chamber by the two walls at $|x|=L_{M} / 2$

$$
W_{\text {rev }}=\left.W\right|_{L_{M} \rightarrow \infty}=A_{2}\left|\alpha^{-1} p_{s}\right|^{2}=\frac{W_{1}}{\alpha}, \quad \tilde{W}=\frac{W_{\mathrm{dc}}}{W_{\text {rev }}}=\frac{1}{\alpha} .
$$

In other words, the contribution toward sound attenuation is the same for the area increase and the sound reverberation within the chamber. No doubt, the reverberating effect derives from the cancellation of sound radiation to the right by the waves reflected from the left junction, vice versa. At the low frequency limit, the reflected wave from the back has very little time delay as it catches up with the radiation in the front. The two have the opposite phase due to the dipole nature of the source.

The plane-wave theory [Eq. (17a)] is presented as filled circles in Fig. 3 and is found to agree with the full numerical simulation perfectly for most of the frequency range. Significant deviation emerges beyond $f=0.5$. This is a somewhat 
surprising agreement considering the fact that the chamber in this example is very short even when compared with the diameter, $L_{M}=1$, and the theory leading to Eq. (17a) assumes plane waves when they meet the junctions at $|x|=L_{M} / 2$. Because of the short chamber used here, the regular pattern of peak to zero insertion loss is not expected to appear for $f$ $<0.5$, beyond which the plane-wave theory would have failed in any case. Longer chamber is used in the experiment and the expected pattern of insertion loss is shown later in Fig. 6(c).

\section{EXPERIMENTAL STUDIES}

In order to validate the predicted broadband reduction in dipole sound radiation from a fan. Experimental studies are conducted in anechoic chamber. Two tests are carried out in the same configuration. In the first, a small loudspeaker of 37 $\mathrm{mm}$ in diameter (not shown) is used to simulate the fan noise source. In the second, a commercially available axial-flow cooling fan is used. An existing expansion chamber, which has a longer chamber length than the configuration studied in Sec. III, is adopted. This configuration is not necessarily the optimal design for practical noise reduction purpose, but it does serve the purpose of illustrating the main noise abatement principle. As is shown below, the spectrum of the insertion loss of this rig has complex patterns, but the experimental result essentially validates the prediction. The following description of the experimental configuration is based on the second test; the configuration for the first test is achieved essentially by the replacement of the fan by the loudspeaker.

\section{A. Configuration and data processing method}

Shown in Fig. 5(a)is the front view of the fan rotor, which has a diameter of $120 \mathrm{~mm}$, a hub-to-tip ratio of 0.5 , nine rotor blades, a tip chord length of $20 \mathrm{~mm}$ and an installation angle of $65^{\circ}$ between the mid-span chord and the rotating plane. The casing has been removed, and the modification is best viewed from the back in Fig. 5(b). The original struts supporting the motor are replaced by a rigid cylinder of $16 \mathrm{~mm}$ in diameter, and a set of nine cylindrical struts of $5 \mathrm{~mm}$ in diameter is attached to the motor-cylinder assembly to enhance the thrust noise radiation at the blade-passing frequency (BPF) and its harmonics. In order to make more noise at frequencies below the BPF, for the sake of enhancing signal-to-noise ratio during test, some cylindrical struts are deliberately displaced to give unequal strut configuration. The fan assembly is held as a cantilever by the rigid cylinder, as shown in Fig. 5(b), and is inserted to the middle of the circular tube of $1457 \mathrm{~mm}$ in total length and $133.5 \mathrm{~mm}$ in diameter.

The baseline configuration of the experiment is illustrated in Fig. 5(c). The fan is installed in the middle of a uniform, finite length tube. The radiated noise is measured at $20 \mathrm{~cm}$ upstream of the open end, and is also off-set by $20 \mathrm{~cm}$ from the center-line. The microphone used is B\&K type 4947, powered by B\&K's Nexus conditioning amplifier type 2693. Another microphone is placed at the same position in the downstream quadrant, which is outside the regime of exit

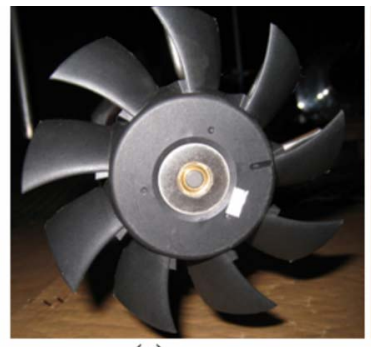

(a)

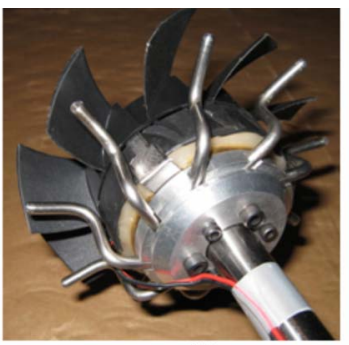

(b)

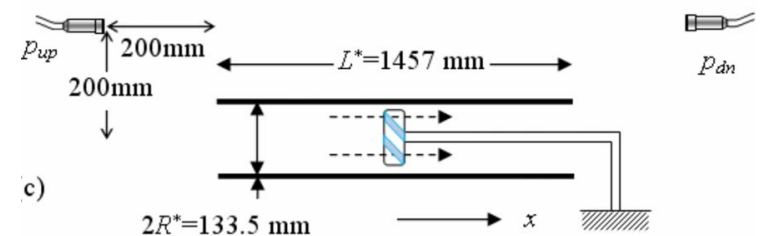

$2 R^{*}=133.5 \mathrm{~mm}$

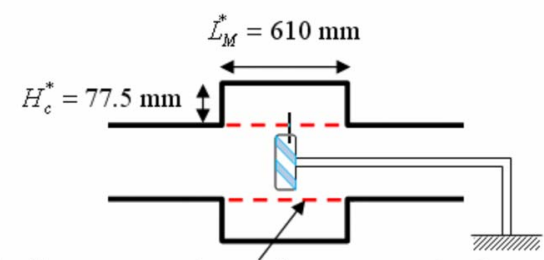

Acoustically transparent inner tube segment, circular

FIG. 5. (Color online) Experimental configurations: (a) the front view of the nine-blade axial-flow cooling fan used; (b) the back view of the rotor-motor assembly attached to a rigid cylinder while nine struts added to create the loud noise from rotor-strut interaction at the coincident mode; (c) the baseline configuration with fan inserted into the middle of a finite circular tube; and (d) expanded circular chamber added around a segment of acoustically transparent inner tube made of $12 \mu \mathrm{m}$ thick plastic membrane wrapping a circular mesh-wire tube of the same diameter as the rest of the inner tube. Configurations (c) and (d) have the same total length with normalized $L_{M}$ $=9.17$, and the cavity depth in (c) is $H_{c}=1.13$.

jet flow. The total length of the tube and the diameter of the main tube are the same as those of the baseline configuration. The middle segment of the tube is replaced by an expansion chamber. The chamber has a length of $610 \mathrm{~mm}$, and the diameter is $288 \mathrm{~mm}$, giving a cavity depth of $H_{c}^{*}$ $=77.5 \mathrm{~mm}$, or a dimensionless $H_{c}=1.16$. When the chamber is sandwiched in between, the upstream and downstream segments of the inner tube is $423.5 \mathrm{~mm}$ long, respectively. In order to keep the flow produced by the fan unchanged, the middle segment of the inner tube is replaced by an acoustically transparent tube of the same diameter, which is shown in Fig. 5(d) as the dashed lines. This is made by wrapping a thin polyvinyl chloride (PVC) membrane of $12 \mu \mathrm{m}$ in thickness around a mesh-wire net formed in cylindrical shape. Note that this PVC membrane is not used in the first test using the small loudspeaker as the source. Flow velocity measurement is made by traversing a hot-wire probe across the inlet section of the tube, and an average flow velocity of $3.5 \mathrm{~m} / \mathrm{s}$ is generated when the fan rotates at $2255 \mathrm{rpm}$ for the baseline configuration shown in Fig. 5(c). The velocity measured for the second configuration with the same fan speed is indistinguishable from that of the baseline with a velocity variation below $0.1 \mathrm{~m} / \mathrm{s}$. It means that the acoustically transparent inner tube in the expanded section is effective in maintaining the same average flow condition. Note that when 
this inner tube is removed, the flow drawn through the whole tube is much smaller due to the apparent added pressure losses at the two junctions.

It may be anticipated that the same rig may not work when the flow through the fan has a high pressure rise. This is so because the air in the cavity must have a single value of pressure, say the average between the two sides, and the cylindrical membrane would be subjected to tensile forces. One side is compressed and another side expanded. Under such tension the acoustic transparency would be compromised. Two solutions are proposed here. First, the application is limited to low pressure-rise applications where the fan design is such that the energy imparted to the fan is mainly in the form of kinetic energy instead of static pressure rise, as is the case for the cooling fan in the current experiment. The second solution is to have a more elaborate cavity design to counter the effect of pressure difference. Suppose the cavity is separated by an impervious membrane at the cross section of the fan center, say $x=0$, and the time-mean pressure at $x$ $<0$ is lower than that in $x>0$. The membrane may be electrically charged and is sandwiched between two rigid but porous plates, which are charged with opposite signs. The membrane is pushed by one plate but pulled by another. In such a push-pull system, the effective spring constant introduced by the two sides also cancels to a large extent. When the membrane is displaced toward the attracting plate, the attraction force increases while the repulsive force reduces with a nearly constant sum. This is an adaptation of commercially available electrostatic loudspeaker design (Baxandall, 1988), for which the charged dielectric membranes are typically made of polyester film with a thickness of 2-20 $\mu \mathrm{m}$, hence little inertia. The porous backing plates would have acoustic damping but that may also be minimized by design. The combination of low inertia, low damping and low stiffness implies good acoustic transparency to the dipole sound generated by the rotor blades. Such a system will be definitely delicate but theoretically possible.

Insertion loss (IL) can be defined by the difference of the sound pressure levels at a given measurement point radiated by the fan installed in the two configurations. When the fan is modeled as a perfect dipole, the out-of-duct radiation through the duct ends is symmetrical in strength with respect to the fan position, $x=0$. Sound radiated from one end can reach the microphone placed on the other side. As an initial validation, it is nevertheless assumed that such cross influence is small and the sound measured at each side of the duct reflects the amount of sound radiation from the same side of the duct. In the prediction code, the boundary condition for the upstream and downstream boundaries "fL" and " $\mathrm{fR}$ " in Fig. 1(b), is changed from the pure outgoing waves in Eq. (5c) to the form of open-end impedance given in Eq. (6). Sound power through the two openings is found by integrating the sound intensity. The sound power level difference $\Delta S W L$ predicted for the two configurations of Figs. 5(c) and 5(d) can be compared with the IL derived from the microphone signals. When there is a physical screen on the fan rotational plane, the two would be the same $(\Delta \mathrm{SWL}=\mathrm{IL})$. In reality, the radiation from the fan is not an ideal dipole. The following theoretical model extracts the dipole part of the fan noise, calculates the insertion loss of the dipole radiation $\mathrm{IL}_{d}$, and then compares it with the predicted $\Delta$ SWL.

Below the cut-on frequency of the duct, the radiated sound approaching the two open ends "fL" and "fR" at $|x|$ $=L^{*} /\left(2 R^{*}\right)=1457 / 133.5=10.9$ is plane wave. Reflected sound is further scattered by the junctions and the fan blades, and joins the sound radiation toward the other open end. Although it is hard to decompose all wave components in such a reverberating environment, as is done in Sec. III for infinite duct with an ideal dipole, it is possible to model the whole middle segment, the fan plus the expansion chamber, as an equivalent source, for which no assumption of its radiation pattern is made. The radiation of the equivalent source is then decomposed into two parts: one with antiphase relation in the upstream and downstream regions, and another with in-phase relation,

$$
p= \begin{cases}\left(p_{m}+p_{d}\right) e^{+i k x}+A e^{i k x}+B e^{-i k x}, & x<-L_{M} / 2 \\ \left(p_{m}-p_{d}\right) e^{-i k x}+A e^{i k x}+B e^{-i k x}, & x>+L_{M} / 2,\end{cases}
$$

where $p_{d}$ and $p_{m}$ denote the amplitudes of the dipole- and monopolelike parts of the field, respectively, $x=0$ is where the source is located, and $A$ and $B$ are the reflections of the equivalent source radiation by the duct ends. Note that Eqs. (19) are not meant to describe the complex field inside the equivalent source occupying $|x| \leq L_{M} / 2$. As stated in Sec. I, there is little true monopole-type source in a low-speed fan like this, and the so-called monopolelike part of the acoustic field is mainly derived from acoustic diffraction by blades and junctions. The reflection coefficient of the open ends is denoted by $\beta$ and is related to the open-end impedance $Z_{\text {open, }}$, as defined in Eq. (6), and it is written together with the definition of the measured transfer function between the two microphones

$$
\beta=\frac{Z_{\text {open }}-1}{Z_{\text {open }}+1}, \quad r_{\text {up }}^{\text {dn }}=\frac{p_{\text {dn }}}{p_{\text {up }}} .
$$

It can be easily shown that

$$
\beta=\frac{B e^{+i k L / 2}}{\left(p_{m}+p_{d}+A\right) e^{-i k L / 2}}=\frac{A e^{i k L / 2}}{\left(p_{m}-p_{d}+B\right) e^{-i k L / 2}} .
$$

When both ends of the duct radiate sound to each microphone such as air pistons, the contribution of the two open ends must be added up. Sound pressure radiated by each air piston is proportional to

$$
\frac{\exp (-i k r)}{r^{2}}\left(x_{\mathrm{ob}}-x_{\mathrm{pist}}\right) n_{x}
$$

where subscript "ob" denotes observer and "pist" for piston, and $n_{x}$ denotes the outward normal direction of the piston. The ratio of radiation from the far-side of the tube of length $L$ to that from the near side is defined as the "cross-radiation" coefficient $\gamma$ and is calculated as follows:

$\gamma=\frac{p_{\text {far }}}{p_{\text {near }}}=-\left(\frac{r}{r_{\text {far }}}\right)^{2} \exp \left[-i k\left(r_{\text {far }}-r_{\text {near }}\right)\right] \frac{L+r \cos \theta}{r \cos \theta}$, 


$$
r_{\text {far }}^{2}=r^{2}+L^{2}+2 r L \cos \theta
$$

where $r$ and $\theta$ are, the distance and angle between the microphone and the near-side duct opening, respectively, and subscript "far" denotes the far-side duct openning. Assuming the transfer function $r_{\text {exit }}^{\text {mic }}$ between the exit pressure and the sound radiated at the microphone position by the near-side opening, the total sound measured at the two microphone positions are

$$
p_{\mathrm{up}}=r_{\mathrm{exit}}^{\mathrm{mic}}\left(p_{\mathrm{fL}}+\gamma p_{\mathrm{fR}}\right), \quad p_{\mathrm{dn}}=r_{\mathrm{exit}}^{\mathrm{mic}}\left(p_{\mathrm{fR}}+\gamma p_{\mathrm{fL}}\right) .
$$

The ratio of sounds measured at the upstream and downstream microphones is related to the sound pressures at the far left (fL) and far right (fR) open ends as follows:

$$
r_{\mathrm{up}}^{\mathrm{dn}}=\frac{p_{\mathrm{dn}}}{p_{\mathrm{up}}}=\frac{p_{\mathrm{fR}}+\gamma p_{\mathrm{fL}}}{p_{\mathrm{fL}}+\gamma p_{\mathrm{fR}}}, \quad \frac{p_{\mathrm{fR}}}{p_{\mathrm{fL}}}=\frac{r_{\mathrm{up}}^{\mathrm{dn}}-\gamma}{1-\gamma r_{\mathrm{up}}^{\mathrm{dn}}}=r^{\prime} .
$$

Expressing the pressures $p_{\mathrm{fL}}$ and $p_{\mathrm{fR}}$ in terms of Eq. (19), such as

$$
p_{\mathrm{fL}}=\left(p_{m}+p_{d}+A\right) e^{-i k L / 2}+B e^{i k L / 2},
$$

the pressure ratio between the two microphones becomes

$$
r^{\prime}=\frac{\left(p_{m}-p_{d}+B\right) e^{-i k L / 2}+A e^{i k L / 2}}{\left(p_{m}+p_{d}+A\right) e^{-i k L / 2}+B e^{i k L / 2}} .
$$

The two equations in Eq. (21), and Eqs. (24) and (25) can be solved for pressure amplitudes $p_{m}, p_{d}, A$, and $B$ in terms of $\beta, r^{\prime}$, and $p_{\mathrm{fL}}$. The latter can be substituted by $p_{\text {up }}$ by solving Eq. (22). Finally, the following decomposition of sounds is obtained:

$$
\begin{aligned}
& p_{d}=\frac{p_{\mathrm{up}}}{r_{\mathrm{exit}}^{\mathrm{mic}}\left(1+\gamma r^{\prime}\right)} \times \frac{\left(e^{i k L}+\beta\right)\left(1-r^{\prime}\right)}{2 e^{i k L / 2}(1+\beta)}, \\
& p_{m}=\frac{p_{\mathrm{up}}}{r_{\mathrm{exit}}^{\mathrm{mic}}\left(1+\gamma r^{\prime}\right)} \times \frac{\left(e^{i k L}-\beta\right)\left(1+r^{\prime}\right)}{2 e^{i k L / 2}(1+\beta)} .
\end{aligned}
$$

Note that the transfer function $r_{\text {exit }}^{\text {mic }}$ can be easily measured by replacing the fan by a loudspeaker; otherwise, the wind screen needed at the duct exit would seriously block the exit. This function should be the same for both ends and for both configurations shown in Figs. 5(c) and 5(d).

Two insertion losses are defined for the experiment, one for the dipole radiation and another for the raw data at upstream measurement point

$$
I L_{d}=20 \log _{10} \frac{\left|p_{d}\right|_{\text {rig } 1}}{\left|p_{d}\right|_{\text {rig } 2}}, \quad I L_{\text {up }}=20 \log _{10} \frac{\left|p_{\text {up }}\right|_{\text {rig } 1}}{\left|p_{\text {up }}\right|_{\text {rig } 2}} .
$$

\section{B. Experimental results}

In the first test, the fan is replaced by a small loudspeaker of $37 \mathrm{~mm}$ in diameter using the same supporting system, as shown in Fig. 5. The loudspeaker is driven by a function generator at discrete frequencies from $120 \mathrm{~Hz}$ up to $1500 \mathrm{~Hz}$ with an interval of $10 \mathrm{~Hz}$. The results of this test are given in Fig. 6, while the corresponding results for the cooling fan as the noise source are given in Fig. 7. In general, the agreement between the prediction and experiment is better for the loudspeaker than that for the fan. Notice that the predicted pattern of insertion loss for the loudspeaker, shown as the dashed line in Fig. 6(c), differs slightly from that for the fan, shown as the dashed line in Fig. 7(c), toward the high frequency region because of the following factors: (i) there is no inertia effect of the separation membrane for the loudspeaker rig; and (ii) the fan noise source is nearer to the wall of the central duct, while the moving diaphragm of the loudspeaker is very close to the center of the duct.

Before analyzing the experimental results, it is instructive to compare the predicted insertion loss for the finite duct, shown as the dashed line in Fig. 6(c), with the ideal insertion loss when the main duct has infinite length, shown as the thin solid line in the same figure and calculated by $I L_{\infty}=10 \log _{10}\left(W_{1} / W\right)$ with power ratio $W_{1} / W$ given in Eq. (17a). As expected from the analysis of Eq. (17c), the peak performance of $I L_{\infty}=13.1 \mathrm{~dB}$ is found at the dc, as well as the full wavelength frequency of $553 \mathrm{~Hz}$. $I L_{\infty}=0$ is found at odd multiples of $277 \mathrm{~Hz}$. The ideal performance at these frequencies coincides with that predicted for the finite duct (thick dashed line). The difference between these two curves reflects the complex open-end reflection.

Focusing now on the experimental data for the loudspeaker test, the spectrum of the extracted dipole radiation $p_{d}$ for the first configuration (no casing treatment) is shown in Fig. 6(a) as the solid line, and that for the second configuration (with expansion chamber) in dashed line, with arbitrary reference value for $0 \mathrm{~dB}$. The lower frequency limit of the test is dictated by the inferior small loudspeaker performance at low frequencies, while the upper limit of the frequency shown is $1500 \mathrm{~Hz}$, which is slightly higher than the first high-order cut-on frequency of $1493 \mathrm{~Hz}$. With very few exceptions, the sound radiation by the second configuration is below that of the first over the bulk of the frequency range.

The raw insertion loss for the upstream measurement point $\mathrm{IL}_{\mathrm{up}}$ is shown in Fig. 6(b) as the open circles, while the prediction for the pure dipole model $\Delta$ SWL is shown in dashed line for comparison. The agreement between them is generally satisfactory although some frequency shifts are found. When the dipole extraction scheme of Eq. (26) is used, the insertion loss defined in Eq. (27) is plotted in Fig. 6(c) as open circles. It has much closer agreement with theoretical prediction (dashed line) based on the pure dipole radiation. The comparison shown in Fig. 6(c) is better than that in Fig. 6(b) both in terms of the overall insertion loss level, around $10 \mathrm{~dB}$, and the spectral pattern.

The experimental results for the fan test are shown in Fig. 7, which is presented in a similar manner as in Fig. 6. The fan noise spectra shown in Fig. 7(a) have sharp peaks due to blade-passing events. The raw insertion loss measured at the upstream microphone $\mathrm{IL}_{\text {up }}$ is shown in Fig. 7(b) as the solid line, which is compared with the theoretical dipole prediction shown in the same figure as the dashed line. There is a broad agreement of trend between the two but there are differences. First, the general value of $\Delta \mathrm{SWL}$ is around 10 $\mathrm{dB}$ while $\mathrm{IL}_{\mathrm{up}}$ is typically about $5 \mathrm{~dB}$. Second, there is significant difference below about $220 \mathrm{~Hz}$, around $550 \mathrm{~Hz}$, and between 1100 and $1300 \mathrm{~Hz}$. These differences are believed to be mainly caused by the deviation of the theoretical model of 

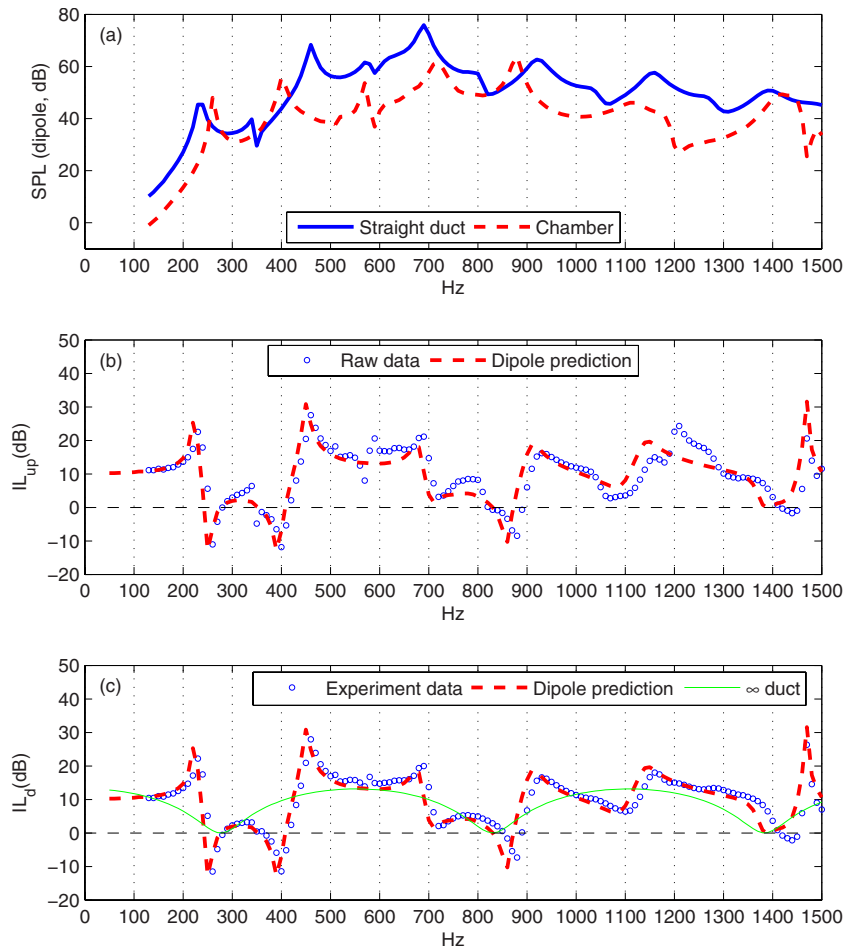

FIG. 6. (Color online) Comparison between prediction and experiment using a loudspeaker of diameter $37 \mathrm{~mm}$ as the dipole source: (a) spectra of the extracted dipole sound radiation; (b) insertion loss $\left(\mathrm{IL}_{\text {up }}\right)$ found at the upstream measurement point (open circles) shown in Fig. 6(c), compared with predicted IL for dipole-only radiation (dashed line); and (c) comparison of measured dipole insertion loss $\mathrm{IL}_{d}$ (open circles), and numerical prediction for the finite rig (dashed line) and the plane-wave prediction for the same chamber with infinite upstream and downstream tubes (thin solid curve).

pure dipole radiation and the real fan. When the dipole extraction scheme of Eq. (26) is used, the insertion loss defined in Eq. (27) has much closer agreement with theoretical prediction based on the pure dipole radiation. The comparison is shown in Fig. 7(c). Both the overall insertion loss level, around $10 \mathrm{~dB}$, and the spectral pattern have been improved. The remaining disagreement is below $100 \mathrm{~Hz}$, between 500 $\mathrm{Hz}$ and $600 \mathrm{~Hz}$, and between $1200 \mathrm{~Hz}$ and $1300 \mathrm{~Hz}$. In both Figs. 7(b) and 7(c), negative insertion loss is rare.

\section{CONCLUSIONS}

This study focuses on the radiation of low frequency noise by a ducted fan. The main numerical results are presented in Fig. 3 for the acoustic configuration of infinite duct, and comparison with experiment is given in Fig. 6 when a loudspeaker is used to simulate the dipole noise radiation from a fan, and in Fig. 7 when a real axial-flow fan is installed in a duct of finite length. The main theoretical explanation is given by the plane-wave theory, which matches rather well with the full numerical simulation at low frequencies. Several conclusions can be drawn.

(i) Sound absorption material is not effective at low frequencies when the lining is shallow. Between the two types of duct lining, bulk reactive lining performs better at low frequencies. When compared with the baseline configuration of no casing treatment, locally reactive lining enhances sound radiation while bulk
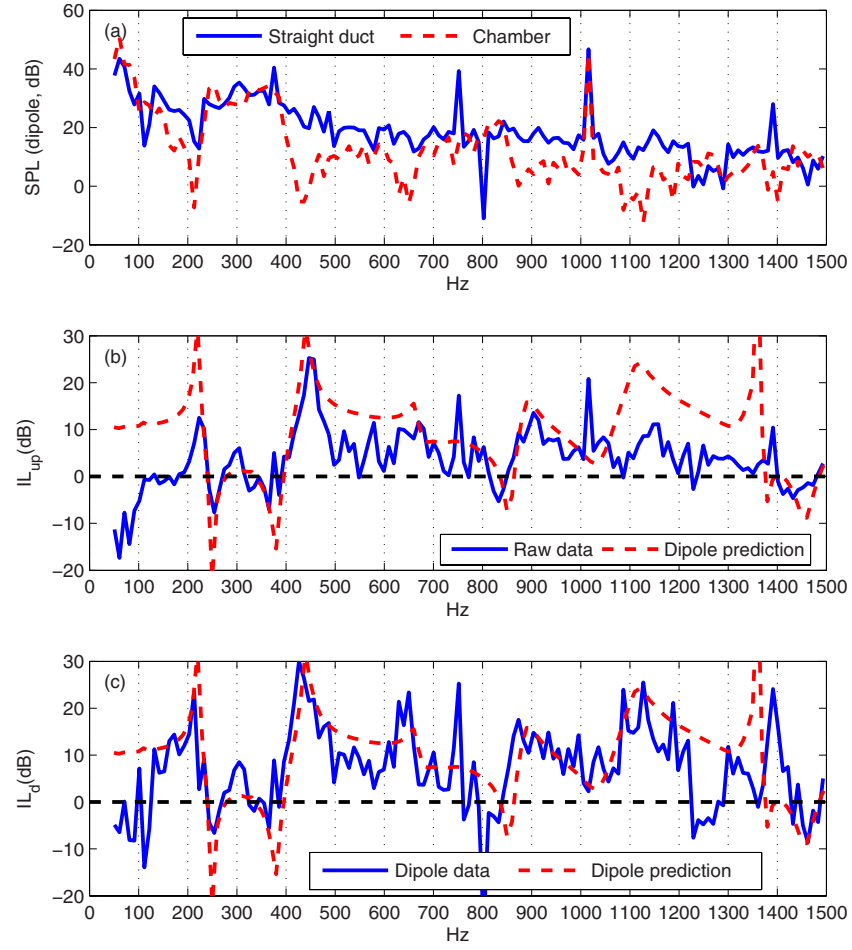

FIG. 7. (Color online) Experimental results for an axial-flow fan: (a) spectra of the extracted dipole sound radiation; (b) insertion loss $\left(\mathrm{IL}_{\text {up }}\right)$ found at the upstream measurement point (solid line) shown in Fig. 5(c), compared with predicted IL for dipole-only radiation (dashed line); and (c) comparison of measured dipole insertion loss $\mathrm{IL}_{d}$ (solid) and theoretical prediction (dashed line).

reactive lining functions as it is intended although there is no absorption toward the dc frequency.

At the extreme low frequencies, an empty casing treatment is the best option. This is essentially an expansion chamber enclosing dipoles. Two mechanisms are at work in reducing noise radiation. One is the reduction in sound intensity by the increased duct diameter, which gives a sound power reduction factor equal to the area ratio, and another is the wave reverberation effect, which incidentally has exactly the same ratio of sound power reduction as the first mechanism.

(iii) The main controlling parameter is the area ratio, while the cavity length is not an important factor as far as the low frequency is concerned. This contrasts sharply with the traditional passive noise abatement, either by dissipative or reactive means. In traditional settings, the volume required tends to infinity when frequency of interest approaches zero. This departure from the traditional design derives from the exploitation of the unique dipole character.

(iv) When the main duct is finite, with both ends open to the environment, standing waves form in the upstream and downstream ducts of the expansion chamber. Complex insertion loss pattern is formed. As far as the chamber itself gives a significant amount of attenuation of fan noise radiation, negative insertion loss is rare. Experimental results using a loudspeaker as the dipole source agree very well with theoretical predic- 
tions. On the other hand, experiment also confirms that the fan noise radiation is not one of pure dipole, but the insertion loss has a pattern that is broadly consistent with theoretical predictions using the dipole model. When the dipole radiation is extracted from the experimental data, very close agreement is achieved with the theoretical prediction over the entire frequency band below the first higher order mode cut-on.

\section{ACKNOWLEDGMENTS}

The authors wish to acknowledge the funding support from the Research Grants Council of the Hong Kong SAR Government under the Grant No. HKU 5170/05E, while part of the computation was supported by HKU 5178/06E. The second author is also supported by a postgraduate studentship by the University of Hong Kong.

Baxandall, P. J. (1988). "Electrostatic loudspeaker," in Loudspeaker and Headphone Handbook, edited by J. Borwick, Butterworths, London, Chap. 3.

Boyd, J. P. (2001). Chebyshev and Fourier Spectral Methods, 2nd ed. (Dover, New York).

Choi, H. L., and Lee, D. J. (2006). "Development of the numerical method for calculating sound radiation from a rotating dipole source in an opened thin duct," J. Sound Vib. 295, 739-752.

Clenshaw, C. W., and Curtis, A. R. (1960). "A method for numerical integration on an automatic computer," Numer. Math. 2, 197-205.

Curle, N. (1955). "The influence of solid boundaries upon aerodynamic sound," Proc. R. Soc. London, Ser. A 231, 505-514.

Delany, M. E., and Bazley, E. N. (1970). "Acoustical properties of fibrous materials," Appl. Acoust. 3, 105-116.

Dunn, M. H., Tweed, J., and Farassat, F. (1999). "The application of a boundary integral equation method to the prediction of ducted fan engine noise," J. Sound Vib. 227, 1019-1048.

Dunne, R. C., and Howe, M. S. (1997). "Wall-bounded blade-tip vortex interaction noise," J. Sound Vib. 202, 605-618.

Eversman, W. (1995). "Theoretical models for duct acoustic propagation and radiation," in Aeroacoustics of Flight Vehicles, Theory and Practice: Noise Control, edited by H. H. Hubbard (Acoustical Society of America, Melville, New York, Vol. 2), Chap. 13.

Ffowcs Williams, J. E., and Hall, L. H. (1970). "Aerodynamic sound generation by turbulent flow in the vicinity of a scattering half plane," J. Fluid Mech. 40, 657-670.

Ffowcs Williams, J. E., and Hawkings, D. L. (1969). "Sound generation by turbulence and by surfaces in arbitrary motion," Philos. Trans. R. Soc. London, Ser. A 264, 321-342.

Fukano, T., Takamatsu, Y., and Kodama, Y. (1986). "The effects of tip clearance on the noise of low pressure axial and mixed flow fans," J. Sound Vib. 105, 291-308.

Goldstein, M. E. (1976). Aeroacoustics (McGraw-Hill International Book Company, New York).

Gutin, L. (1948). "On the sound field of a rotating propeller," Zh. Tekh. Fiz. 6, 899-909.

Hill, S. D., Elder, R. L., and McKenzie, A. B. "Application of casing treat- ment to an industrial axial-flow fan," Proc. Inst. Mech. Eng., Part A 212 225-233 (1998).

Huang, L. (2003). "Characterizing computer cooling fan noise," J. Acoust. Soc. Am. 114, 3189-3200.

Huang, L. (2008). "Membrane covered duct lining for high frequency noise attenuation: Prediction using a Chebyshev collocation method," J. Acoust. Soc. Am. 124, 2918-2929.

Huang, L., and Cheung, S. C. (2008). "Computation of sound radiation in a duct by Chebyshev collocation," in Proceedings of the INTER-NOISE 2008, Paper No. 0512.

Jang, C. M., Fukano, T., and Furukawa, M. (2003). "Effects of the tip clearance on vortical flow and its relation to noise in an axial flow fan," JSME Int. J. Ser. B 46, 356-365.

Karniadakis, G. E., and Sherwin, S. (2005). Spectral/hp Element Methods for Computational Fluid Dynamics, 2nd ed. (Oxford University Press, New York).

Kemp, N. H., and Sears, W. R. (1953). "Aerodynamic interference between moving blade rows," J. Aeronaut. Sci. 20, 583-598.

Kemp, N. H., and Sears, W. R. (1955). "Unsteady forces due to viscous wakes in turbomachines," J. Aeronaut. Sci. 22, 478-483.

Lauchle, G. C., Macgillivray, J. R., and Swanson, D. C. (1997). "Active control of axial-flow fan noise," J. Acoust. Soc. Am. 101, 341-349.

Lewy, S. (2007). "Prediction of turbofan rotor or stator broadband noise radiation," Acta Acust. Acust. 93, 275-283.

Lighthill, M. J. (1952). "On sound generated aerodynamically. I. General theory," Proc. R. Soc. London, Ser. A 211, 564-587.

McAlpine, A., Astley, R. J., Hii, V. J. T., Baker, N. J., and Kempton, A. J. (2006). "Acoustic scattering by an axially-segmented turbofan inlet duct liner at supersonic fan speeds," J. Sound Vib. 294, 780-806.

Morse, P. M., and Feshbach, H. (1953) Method of Theoretical Physics Part II (McGraw-Hill, New York).

Morse, P. M., and Ingard, K. U. (1968) Theoretical Acoustics (McGraw-Hill, New York).

Motsinger, R. E., and Kraft, R. E. (1995). "Design and performance of duct acoustic treatment," in Aeroacoustics of Flight Vehicles, Theory and Practice: Noise Control, edited by H. H. Hubbard, Acoustical Society of America, Melville, New York, Vol. 2, Chap. 14.

Namba, M., and Murahashi, A. (2008). "Aeroacoustic control of fan tone noise," AIAA J. 46, 54-60.

Neuhaus, L., Schulz, J., Neise, W., and Moser, M. (2003). "Active control of the aerodynamic performance and tonal noise of axial turbomachines," Proc. Inst. Mech. Eng., Part A 217, 375-383.

Pierce, A. D., Cleveland, R. O., and Zampollia, M. (2002). "Radiation impedance matrices for rectangular interfaces within rigid baffles: Calculation methodology and applications," J. Acoust. Soc. Am. 111, 672-684.

Prezelj, J., and Čudina, M. (2007). "Dipole in orthogonal direction as a secondary source for active noise control in ducts," Acta Acust. Acust. 93, 63-72.

Schulten, J. B. H. M. (2001). "Active control of rotor-stator interaction noise through vibrating vanes," AIAA Paper No. 2001-2151.

Trunzo, R., Lakshminarayana, B., and Thompson, D. E. (1981). "Nature of inlet turbulence and strut flow disturbances and their effect on turbomachinery rotor noise," J. Sound Vib. 76, 233-259.

Tyler, J. M., and Sofrin, T. G. (1962). "Axial flow compressor noise studies," SAE Trans. 70, 309-332.

Wang, J., and Huang, L. (2006). "Active control of drag noise from a small axial flow fan," J. Acoust. Soc. Am. 120, 192-203.

Wang, J., Huang, L., and Cheng, L. (2005). "A study of tonal noise control for a small axial flow fan,” J. Acoust. Soc. Am. 117, 734-743. 